\title{
A atividade física como ferramenta de apoio às ações da Atenção Primária à Saúde
}

\section{Physical Activity as primary health care support tool}

Flávio Renato Barros da Guarda

Rafaela Niels da Silva²

Suzeanny Magna da Silva

Paulo Roberto de Santana ${ }^{3}$

\section{RESUMO}

O objetivo deste trabalho é apresentar as ações de atividades físicas desenvolvidas pela equipe dos Núcleos de Apoio à Saúde da Família (NASF) no município de Igarassu (Pernambuco). Destacam-se as ações articuladas com a rede de saúde municipal: aulas regulares de ginástica, atividades educativas para grupos especiais, estímulo às práticas corporais regionais e formação de grupos de caminhada, além de ações compartilhadas com as equipes de saúde da família. Articulações intersetoriais são desenvolvidas em parceria com Centros de Convivência de Idosos, ONGs e outros espaços sociais. O trabalho da equipe NASF de Igarassu baseia-se nas diretrizes estabelecidas pelo Ministério da Saúde e nas políticas nacionais da atenção básica e de promoção da saúde, entendendo a integralidade, participação social e intersetorialidade como ferramentas para a consecução dos princípios da promoção da saúde e capacitação dos sujeitos para intervir sobre aspectos individuais e coletivos da saúde.

\section{PALAVRAS-CHAVE}

Promoção da Saúde, Atividade Física, Sistema Único de Saúde.

\begin{abstract}
The aim of this paper is to present physical activities actions developed by NASF (primary care support team in public bealth) in Igarassu city, Brazil. We can detach partnership actions developed in conjunction with the municipal health, as regular exercises sections, educative activities for special groups, stimulus to the regional body practices and walking groups, and actions shared with primary bealth care teams. Intersectoral joints are developed in partnership with the Elderly Centres, NGOs and other social spaces. NASF team job in Igarassu is based on oficial guidelines of brazilian bealth ministry and primary care and health promotion policies and understand the full social participation and intersectoral as tools for the achievement of the principles of health promotion training subjects to speak on aspects of individual and collective bealth.
\end{abstract}

\section{KEYWORDS}

Physical Activity; Health Promotion; Unified Health System. 


\section{INTRODUCCÃO}

Visando ampliar o escopo das ações da Atenção Primária em Saúde (APS) e garantir o princípio da integralidade do cuidado nas ações de promoção, proteção, diagnóstico e recuperação, o Ministério da Saúde brasileiro criou, desde 2008, os Núcleos de Apoio à Saúde da Família (NASF)ํ․ As atividades do NASF estão divididas em nove áreas estratégicas: práticas integrativas e complementares; reabilitação; alimentação e nutrição; saúde mental; serviço social; saúde da criança, do adolescente e do jovem; saúde da mulher, assistência farmacêutica e atividade física/praticas corporais² ${ }^{2}$ Os núcleos podem ser compostos por diversas categorias profissionais da saúde, entre as quais, os profissionais de educação física ${ }^{1}$.

Conhecendo os benefícios da prática regular de atividades físicas sobre a saúde de diversos grupos populacionais ${ }^{3,4,5}$ e visando estimular a adoção de um estilo de vida mais ativo entre seus munícipes, a secretaria de saúde de Igarassu, municipio da Região Metropolitana do Recife, Pernambuco, implantou dois NASF em seu território no ano de 2010, incoporando profissionais de eduação física a essas equipes.

As atividades físicas/práticas corporais desenvolvidas pelas equipes do NASF Igarassu são realizadas de acordo com as diretizes estabelecidas pelo Ministério da Saúde ${ }^{2}$ e visam estimular a adoção e manutenção de comportamentos fisicamente ativos nos domínios das atividades domésticas, do lazer e do deslocamento.

\section{FUNCIONAMENTO}

As atividades físicas do NASF Igarassu são desenvolvidas por dois profissionais de educação física, sendo um com especialização em saúde pública. Utilizam-se espaços como Unidades de Saúde da Família (USF) praças, igrejas e centros comunitários.

A equipe realiza visitas regulares às Unidades de Saúde da Família, desenvolvendo ações educativas sobre a importância da prática de atividades físicas para diversos grupos populacionais, tais como gestantes, hipertensos e diabéticos, além de estimular práticas corporais regionais e a formação de grupos de caminhada. Busca, ainda, estabelecer parcerias com o centro de convivência de idosos do município, associações de moradores e igrejas locais, de modo a aumentar os espaços de discussão dos benefícios e incentivo à prática de atividades físicas.

Com o apoio das secretarias de comunicação e educação, e do Conselho de Idosos do município, os profissionais de educação física do NASF Igarassu realizam atividades regulares junto às Unidades de Saúde da Família (USF) do território, ONG SOS Brasil Aldeias Infantis, Centro de Convivência de Idosos e outros espaços sociais. Promovem encaminhamentos para o programa de atividades físicas do departamento de esportes da secretaria de educação, divulgação dos eventos e datas comemorativas, apoio às ações do Programa Saúde na Escola, além de atuarem como mediadores das discussões e intervenções sobre a importância da adoção/manutenção de rotinas de lazer, brincadeiras e deslocamentos ativos.

O NASF Igarassu ainda oportuniza a realização de estágios para estudantes de duas instituições privadas de ensino superior, permitindo que acadêmicos 
dos cursos de Educação Física, Nutrição, Enfermagem e Psicologia adquiram habilidades e conhecimentos sobre os NASF e promoção da saúde e da atividade física dentro do Sistema Único de Saúde.

QUADRO 1 - Atividades desenvolvidas no NASF Igarassu

\begin{tabular}{|c|c|c|c|c|}
\hline Atividades & Local & $\mathrm{N}$ & Frequência semanal & Articulações e Parcerias \\
\hline Aulas de Ginástica & Unidades de Saúde da Família & 60 & $\begin{array}{l}\text { Segundas, quartas e sextas- } \\
\text { feiras das 08:00 às 10:00 e das } \\
\text { 13:00 às 15:00 horas }\end{array}$ & $\begin{array}{l}\text { Equipes de Saúde da Família e Igrejas } \\
\text { do território }\end{array}$ \\
\hline $\begin{array}{l}\text { Atividades de } \\
\text { Grupo latividades } \\
\text { educativas; roda } \\
\text { de conversa. CINE } \\
\text { SAÚDE) }\end{array}$ & $\begin{array}{l}\text { Unidades de Saúde de Família } \\
\text { e Território }\end{array}$ & 150 & $\begin{array}{l}\text { Terças e quintas-feiras das } \\
08: 00 \text { às 12:00 e das 13: } 00 \text { às } \\
17: 00\end{array}$ & $\begin{array}{l}\text { Igrejas; } \\
\text { Associações de moradores; grupos de } \\
\text { jovens; } \\
\text { Equipes de Saúde da Família e demais } \\
\text { profissionais do NASF }\end{array}$ \\
\hline $\begin{array}{l}\text { Danças, jogos } \\
\text { cooperativos e de } \\
\text { salão }\end{array}$ & $\begin{array}{l}\text { Centro de Convivência de } \\
\text { Idosos }\end{array}$ & 40 & $\begin{array}{l}\text { Quartas e sextas-feiras das } \\
\text { 06:00 às 07:00 horas }\end{array}$ & $\begin{array}{l}\text { Unidade de Saúde da Família Beira } \\
\text { Mar I; } \\
\text { Conselho de Idosos de Igarassu }\end{array}$ \\
\hline $\begin{array}{l}\text { Jogos Populares e } \\
\text { cooperativos }\end{array}$ & Aldeias Infantis & 60 & $\begin{array}{l}\text { Segundas e quartas-feiras das } \\
11: 00 \text { às } 12: 00\end{array}$ & ONG SOS Brasil Aldeias Infantis \\
\hline $\begin{array}{l}\text { Apoio às atividades } \\
\text { de Educação } \\
\text { Promoção da Saúde } \\
\text { desenvolvidas pela } \\
\text { ESF }\end{array}$ & $\begin{array}{l}\text { Todas as Unidades de Saúde } \\
\text { da Família do Município }\end{array}$ & 150 & $\begin{array}{l}\text { Terças e quintas-feiras à tarde, } \\
\text { em horários variados }\end{array}$ & $\begin{array}{l}\text { Gerências de Territórios; Associações } \\
\text { Comunitárias; } \\
\text { Igrejas; } \\
\text { Equipes de Saúde da Família; demais } \\
\text { profissionais do NASF. }\end{array}$ \\
\hline
\end{tabular}

A Secretaria de Saúde proporciona o apoio institucional às atividades do NASF, como: carros de som e apoio para eventos regulares e temáticos; médicos, enfermeiros e técnicos de enfermagem para atividades permanentes e eventos, dentre outros. Outros insumos, bem como a complementação do pagamento dos profissionais e arte-educadores constituem a contrapartida do município, o qual forneceu a sala de reunião e o material permanente (computador, impressora, entre outros) para a realização de atividades de planejamento do NASF, devido às limitações de espaço físico de algumas unidades de saúde.

\section{IMPLANTAC̄ÃO E AVALIAC̄̃̃O DO NASF}

Antes da implantação dos Núcleos, a equipe da secretaria de saúde realizou o mapeamento e diagnóstico da área, conforme previsto na Política Nacional da Atenção Básica ${ }^{6}$. Além disso, discutiu estratégias de empoderamento da população a ser atendida e ferramentas que pudessem auxiliar tanto na implanta- 
ção, quanto na avaliação do NASF Igarassu, de modo a assegurar o controle e a participação social nas ações ${ }^{7}$.

O planejamento das ações foi discutido e pactuado entre trabalhadores, gestores, parceiros e população local, de modo a identificar e ponderar as diferenças de prioridades das partes interessadas. Em seguida, elaborou-se o modelo lógico do NASF (figura 1), com informações pertinentes ao contexto da população a ser assistida.

\section{INSUMOS}

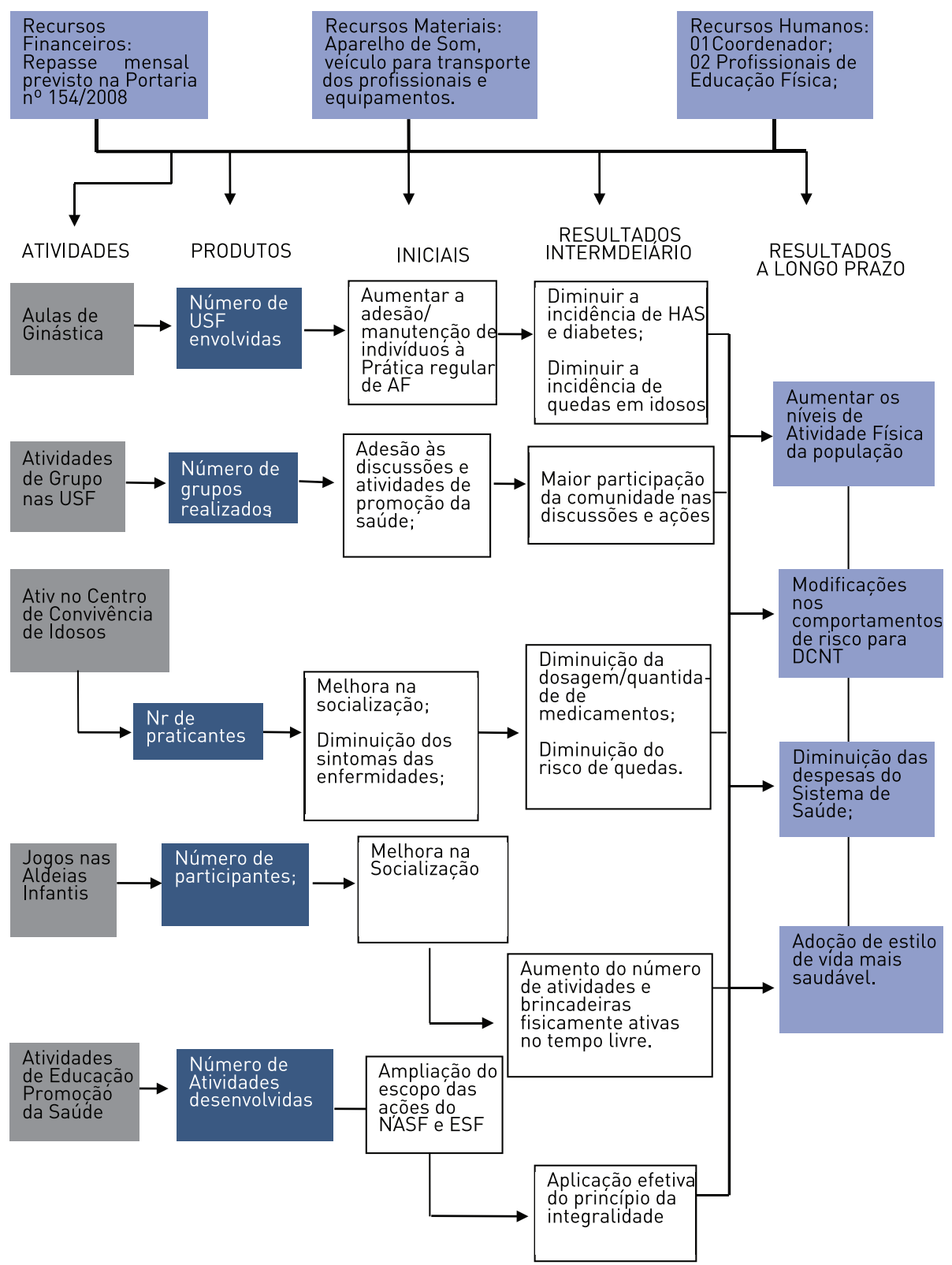

FIGURA 1 - Modelo Lógico do NASF Igarassu

O modelo foi elaborado sob a lógica prospectiva ${ }^{8}$, iniciando-se da apresentação dos insumos e atividades. Estes foram então vinculados aos produtos e resultados (iniciais, intermediários e de longo prazo) esperados, de modo a permitir a avaliação. 
O Modelo Lógico de avaliação das atividades físicas/práticas corporais do NASF Igarassu (Figura 1) descreve os recursos pessoais, materiais e financeiros ( para a execução das, as quais incluem aulas de ginásticas ministradas nas Unidades de Saúde da Família do território do NASF.

Os profissionais de Educação Física ainda desenvolvem atividades educativas e rodas de conversa sobre temas relacionados a atividades físicas/práticas corporais, além de conduzirem parte das ações do Programa Cine Saúde, o qual discute problemas de interesse geral da comunidade a partir da exibição de vídeos, filmes de ficção e documentários.

O modelo Lógico considera como o número de indivíduos ou unidades atendidos por cada atividade, e os esperados variam desde a perspectiva de uma maior participação da comunidade nas atividades do NASF, na fase inicial, até diminuição da exposição a fatores de risco para Doenças e Agravos Não Transmissíveis (DCNT) em alguns anos.

\section{DISCUSSÃO}

As atividades físicas/práticas corporais desenvolvidas pelos profissionais do NASF Igarassu apoiam-se nas diretrizes do Ministério da Saúde ${ }^{2}$ e na Política Nacional de Promoção a Saúde 9 . Esta última compreende que as Práticas Corporais são expressões individuais e coletivas do movimento humano, advindo do conhecimento e da experiência em torno do jogo, da dança, do esporte, da luta, da ginástica. Logo, as ações desenvolvidas nos níveis individual, coletivo e em grupos populacionais específicos, bem como em variados contextos parecem corroborar com as propostas como as de Doyle e Ward $(2001)^{10}$ e Buss $(2000)^{11}$. Todas as ações são planejadas com base no entendimento de que as estratégias de incentivo à adoção de estilos de vida mais ativos demandam abordagens de caráter social e institucional, além de intervenções legislativas, ambientais, organizacionais e institucionais, buscando propor e reforçar articulações com diversos atores sociais e esferas de governo.

O NASF Igarassu busca incorporar suas atividades às agendas da Atenção Primária à Saúde e demais ações e serviços do SUS municipal, em cumprimento ao previsto nas políticas estratégicas do campo da saúde ${ }^{6,9}$, sobretudo mediante estabelecimento de parcerias com entidades governamentais e não-governamentais.

Embora parte das atividades físicas/práticas corporais do NASF Igarassu sejam desenvolvidas em parceria com as Equipes de Saúde da Família e do NASF, os profissionais de Educação Física que nele atuam relatam dificuldades de articular ações e de organizar processos de trabalho com as demais categorias da saúde. Isso de certa forma compromete a efetivação do princípio da integralidade e a utilização das ferramentas de apoio do NASF como clínica ampliada, projetos terapêuticos singulares e de saúde do território. Souza e Loch $(2011)^{12}$ relataram problema similar ao investigar a atuação de profissionais de educação física no norte do estado do Paraná, levando-nos a questionar a possível necessidade de investimentos em formação de base e continuada de modo a atender a essa importante demanda do trabalho na Atenção Primária.

\section{CONSIDERAÇÕES FINAIS}

Baseando-se nas diretrizes específicas de funcionamento e nas políticas nacio- 
nais da atenção básica e de promoção da saúde, o NASF Igarassu busca na integralidade, na participação social e na intersetorialidade as ferramentas para a consecução dos princípios da promoção da saúde, visando desenvolver ações no território que capacitem os sujeitos a intervir sobre aspectos individuais e coletivos da saúde, da divisão de responsabilidades com outros atores e da cogestão.

O desenvolvimento de um modelo lógico de avaliação para as atividades físicas/práticas corporais visa verificar o potencial de efetividade das estratégias de incentivo à adoção de estilos de vida ativos, de modo a identificar e analisar as ligações entre seus recursos, atividades, produtos e resultados esperados.

\section{Contribuições}

Flávio da Guarda sugeriu o tema, orientou e participou da redação do manuscrito. Os demais autores contribuíram para a redação e revisão final do texto.

\section{REFERÊNCIAS}

1. Brasil. Ministério da Saúde. Portaria n ${ }^{\circ}$ 154, de 24 de janeiro de 2008. Cria os Núcleos de Apoio à Saúde da Família - NASF. Diário Oficial [da] República Federativa do Brasil. Brasília, DF, 25 jan. 2008. Seção 1. p. 47-50.

2. Brasil. Ministério da Saúde. Secretaria de Atenção à Saúde. Departamento de Atenção Básica. Diretrizes do Nasf / Ministério da Saúde, Secretaria de Atenção à Saúde, Departamento de Atenção Básica. - Brasília: Ministério da Saúde, 2009.

3. Fernandes RA, Christofaro DG, Casonatto J, Codogno JS, Rodrigues EQ, Cardoso ML, Kawaguti SS, Zanesco A. Prevalence of dyslipidemia in individuals physically active during childhood, adolescence and adult age. Arq Bras Cardiol 2011;97(4):317-323.

4. Fernandes RA, Zanesco A. Early physical activity promotes lower prevalence of chronicle diseases in adulthood. Hypertens Res 2010;33:(9)926-931.

5. Donnelly JE, Blair SN, Jakicic JM, Manore MM, Rankin JW, Smith BK; American College of Sports Medicine. American College of Sports Medicine Position Stand. Appropriate physical activity intervention strategies for weight loss and prevention of weight regain for adults. Med Sci Sports Exerc 2009;41(2):459-471.

6. BRASIL. Ministério da Saúde. Portaria MS/GM n 2.488, de 21 de outubro de 2011. Aprova a Política Nacional de Atenção Básica. Diário Oficial da União, Poder Executivo, Brasília, DF, Seção 1, p.48, 24 out. 2011.

7. Brasil. Lei no 8.142 , de 28 de setembro de 1990: dispõe sobre a participação da comunidade na gestão do Sistema Único de Saúde e sobre as transferências intergovernamentais de recursos financeiros na área da saúde e dá outras providências. Brasília: Congresso Nacional; 1990.

8. U.S. Department of Health and Human Services. Physical Activity Evaluation Handbook. Atlanta, GA. EUA. U.S. Department of Health and Human Services, Centers for Disease Control and Prevention; 2002.

9. Ministério da Saúde. Secretaria de Vigilância em Saúde. Secretaria de Atenção à Saúde. Política nacional de promoção da saúde / Ministério da Saúde, Secretaria de Vigilância em Saúde, Secretaria de Atenção à Saúde. - Brasília : Ministério da Saúde, 2006.

10. Doyle E, Ward, S. The process of community health education and promotion. Mayfield Publishing Company: Mountain View, 2001.

11. Buss, PM. Promoção da saúde e qualidade de vida. Rev. C S Col. 2000; 5 (1): 163-177.

12. Souza SC, Loch MR. Intervenção do profissional de educação física nos Núcleos de Apoio à Saúde da Família em municípios do norte do Paraná. Revista Brasileira de Atividade Física e Saúde 2011; 16 (11): 5-10.

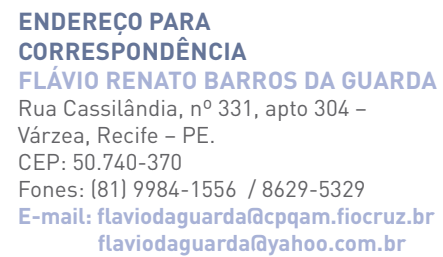

